\title{
Estrategias didácticas en la enseñanza- aprendizaje: lúdica y retroalimentación en el estudio de Química conceptual en alumnos de la Escuela Preparatoria Regional de Atotonilco
}

Didactic strategies in teaching-learning: playful and feedback in the study of conceptual chemistry in students of Atotonilco High School

\author{
Eduardo Zaragoza Ramos ${ }^{1}$ \\ Ronilson Freitas de Souza ${ }^{2}$ \\ Sandra Luz Díaz Díaz ${ }^{3}$ \\ Diógenes Hernández Espinoza ${ }^{4}$ \\ Rosa Marisela Villalobos Díaz ${ }^{5}$ \\ Luz Teresa Olmos Durán ${ }^{6}$ \\ Edgar Eduardo Orozco Ávila7 \\ Reginaldo González Téllez ${ }^{8}$ \\ Carmen Gisela Villalobos Vázquez ${ }^{9}$ \\ Margarita Anahí Ocegueda Camacho ${ }^{10}$
}

\section{Resumo}

En este artículo se describe una actividad de inicio de ciclo en la unidad de aprendizaje Química I. Se evalúa la influencia que dos factores experimentales, lúdica y retroalimentación, ejercen en el rendimiento académico de los estudiantes de la Escuela Preparatoria Regional de Atotonilco. La finalidad es diseñar, aplicar y evaluar una estrategia de enseñanza-aprendizaje que logre que el educando se apropie de los conceptos y términos que en esta ciencia se abordan. Para ello se propone que busquen un paquete conteniendo una lectura fragmentada. Una vez que localizan el paquete, en equipo, deben estructurar la lectura y realizar un mapa conceptual a partir de la misma. A continuación el profesor retroalimenta el contenido temático y posteriormente se aplica un cuestionario a cada individuo para evaluar el nivel de aprovechamiento de los contenidos temáticos. Los datos obtenidos se analizaron en un diseño experimental con dos factores, lúdica y retroalimentación. Los resultados muestran que estos factores incrementan el aprovechamiento de los contenidos temáticos. Con la lúdica los estudiantes

\footnotetext{
Escuela Preparatoria Regional de Atotonilco, Universidad de Guadalajara | eduardo.zramos@academicos.udg.mx Universidade do Estado do Pará | ronilson@uepa.br

Escuela Preparatoria Regional de Atotonilco, Universidad de Guadalajara | sandraluz@sems.udg.mx Universidad de Talca | dhernandez@utalca.cl

Escuela Preparatoria Regional de Atotonilco, Universidad de Guadalajara | marivilladiaz@hotmail.com Escuela Preparatoria Regional de Atotonilco, Universidad de Guadalajara | tere334@hotmail.com Escuela Preparatoria Regional de Atotonilco, Universidad de Guadalajara | tivi20@hotmail.com Escuela Preparatoria Regional de Atotonilco, Universidad de Guadalajara | regisglezt@hotmail.com Escuela Preparatoria Regional de Atotonilco, Universidad de Guadalajara | gigiviva@hotmail.com

${ }_{10}$ Escuela Preparatoria Regional de Atotonilco, Universidad de Guadalajara | oc.anahi@gmail.com
} 
incrementaron sus calificaciones alrededor de 30 puntos, mientras que se logra un aumento de aproximadamente 15 puntos cuando se aplica la retroalimentación.

Palabras clave: estrategia lúdica; retroalimentación; competencias; química general; enseñanza-aprendizaje.

\section{Abstract}

In this article is described the development of a cycle beginning activity in Chemistry 1 subject. In it, are evaluated two experimental factors, ludic and feedback, and the effect than these exercises on the academic performance on student from the Escuela Preparatoria Regional de Atotonilco. The aim is to design, apply and evaluate a teaching-learning strategy that enables the students to appropriate the concepts and terms that are analyzed in this science and for this purpose it is proposed that they conduct the search for a package containing a cut reading in fragments. Once they locate the package, as a team, they must structure the reading and make a conceptual map from it. In a subsequent session, the teacher makes a feedback about the reading and the conceptual map and later a questionnaire is applied to each individual to evaluate the level of achievement of the thematic contents. The obtained data were introduced in an analysis of variance for an experimental design with two factors, ludic and feedback. The results show that the application of these factors is advisable, since in the treated groups the understanding of thematic contents was better, with respect to when it was not applied. With the ludic was achieved an increase in the scores students of around 30 points, while achieving an increase of approximately 15 points when the feedback is applied.

Keywords: playful strategy; feedback; competencies; general chemistry; teaching and learning.

\section{Introducción}

Para construir conocimientos se deben ordenar datos y códigos de manera que queden todos estructurados. La comprensión implica la interpretación correcta de los conceptos de cada objeto de estudio, y entre más logre familiarizarse el estudiante con el lenguaje en cuestión, mayor será su comprensión de contenidos temáticos subsecuentes (Córdova, 1994).

Ausubel en su teoría del conocimiento sugiere que un individuo retiene conocimientos sólo si estos le son significativos (Ausubel y col., 1976). Además Ontoria (2000) y Moreira (1998), mencionan que la adquisición de conocimientos es semejante a un juego de rompecabezas en el que habremos de introducir piezas que encajen perfectamente en lo que ya se ha construido, es decir, lo que deseemos que nuestros estudiantes aprendan debe relacionarse con lo que ya conocen. Si los conocimientos nuevos no conectan en lo ya existente, el estudiante no lo retendrá debido a que no le encuentra importancia, según lo que ya sabe.

Ese proceso de reconstrucción que ocurre durante el aprendizaje significativo es semejante al proceso de armar mapas conceptuale, instrumentos que se emplean para organizar y representar el conocimiento (Castillo, 2009). Según Dürsteler (2004) su objetivo es representar relaciones existentes entre conceptos en forma de preposiciones. "Los conceptos están incluidos en figuras geométricas, mientras que las relaciones entre ellos se explicitan mediante líneas que unen sus formas 
respectivas. Las líneas, a su vez, tienen palabras conectivas que describen cuál es tipo de relación que une los conceptos" (Castillo 2009, 45).

Es una técnica que ayuda a percatarte de tu conocimiento, implica darte cuenta de lo que sabías y lo que no sabías, así como la manera en que ambos se relacionan. Demuestran las habilidades cognoscitivas, afectivas y sociales que el estudiante ha logrado. Ayudan a estructurar ideas y procedimientos para construir nuevos conocimientos, favorecen el desarrollo de la habilidad del lenguaje y de la creatividad (Castillo, 2009). Además permite conocer el significado que los conocimientos tienen para cada estudiante a partir de las relaciones y estructura con que construyen el esquema (Novak, 1997).

Por otra parte, está comprobado que la actividad física, asumida como juego o lúdica, divierte y forma al individuo. Correr, saltar y jugar en continuo movimiento estimula las funciones cerebrales y provoca momentos agradables que a la postre favorecen el rendimiento cognitivo en el sujeto (Drobnic y col., 2013).

La lúdica, motiva al estudiante, promueve la creatividad, imaginación, aprendizaje espontaneo y desarrolla el pensamiento abstracto (Henricks, 1999; Cannoy Newble, 2000; Lieberman, 1977; Vygotsky, 1978; Bruner, 1986; Piaget e Inhelder, 1997; Franco, 2014, Zaragoza y col., 2016).

Así mismo la retroalimentación, por su parte, es una herramienta que permite apoyar al estudiante en su proceso de adquisición de los conocimientos (Lózano y Tamez, 2014). Además, como lo demuestran algunos estudios, (Holmes y Papageorgiou, 2009; Lipnevich y Smith 2009), ésta es considera de gran importancia por los alumnos y expresan que una buena retroalimentación por parte del docente permite que ellos logren el objetivo del curso.

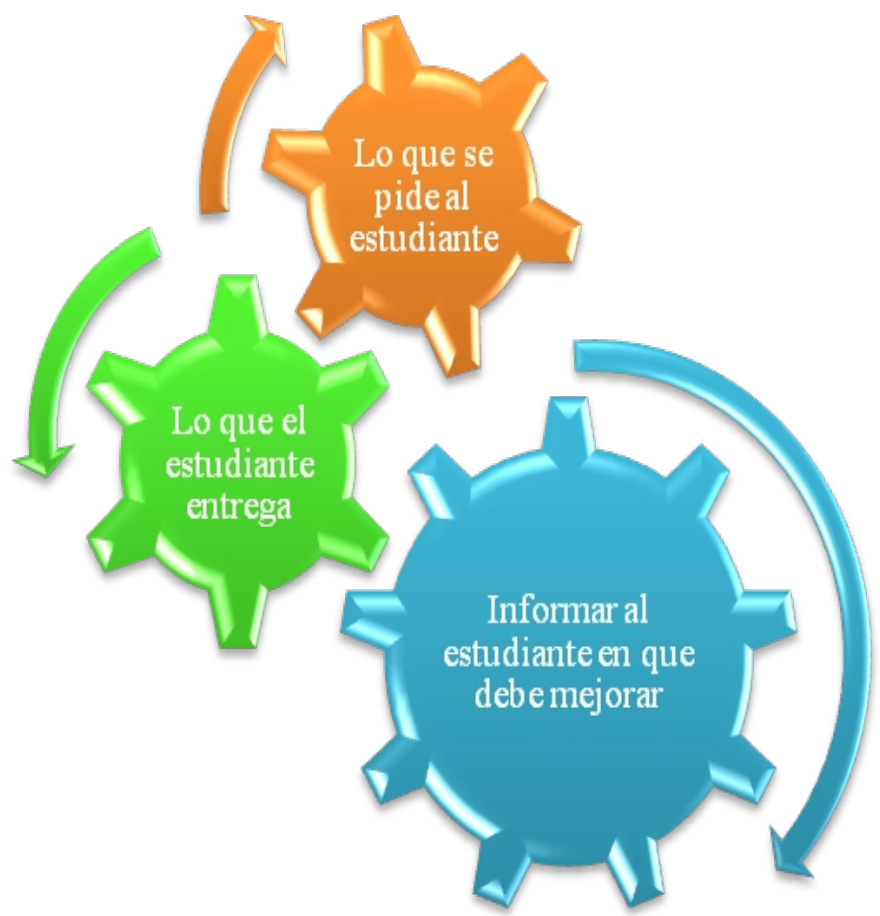

Imagen 1. Proceso para decidir qué mensaje se debe escribir al alumno al retroalimentar (Modificado de Lózano y Tamez, 2004). Fuente propia. 
Según Lózano y Tamez (2004), no basta con decir al estudiante -estás mal-. Hay que informarle cuáles son sus áreas de oportunidad para que pueda mejorar y de esa manera logre una mejor construcción del conocimiento (imagen 1). La retroalimentación debe volver consciente al alumno de lo que entregó y lo que debió haber entregado, debe ayudarle a comprender que le hace falta para cumplir el objetivo del curso (Hattie y Timperley, 2007).

Ahora bien, con la finalidad de contextualizar nuestra investigación, se describe el sistema educativo en México, que se divide de la siguiente manera (SEP, 2011):

1. Educación básica

\subsection{Kínder}

1.2. Escuela primaria

1.3. Escuela secundaria

2. Educación Media Superior (EMS)

3. Educación Superior (ES)

La Universidad de Guadalajara (UdG) es una institución que ofrece estudios de EMS y ES. El nivel medio, también conocido como bachillerato, se cursa en escuelas preparatorias, ya sean regionales; fuera de la Ciudad de Guadalajara, o metropolitanas; dentro de la ciudad. Este estudio se realizó en la Escuela Preparatoria Regional de Atotonilco, cuyo nombre proviene de la población en la que se encuentra.

Nuestra escuela, al igual que todas las preparatorias de la UdG, desde el año 2008, ha modificado su programa educativo y ha migrado a estudios basados en competencias, tomando el nombre de Bachillerato General por Competencias (UDG, 2008). Esta modificación ha provocado que las planeaciones didácticas sean no tradicionalistas, que el estudiante domine destrezas y habilidades que lo formen competente para solucionar situaciones en la vida diaria (Sacristán y col., 2009).

Los retos descritos, provocan que los docentes se encuentren en constante formación y en la creación de estrategias de enseñanza-aprendizaje novedosas que promuevan en el estudiante el desarrollo de competencias, siendo la lúdica una de las herramientas aplicadas. Se señala además que en unidades de aprendizaje de carácter científico, es más notoria dicha tendencia (Zaragoza y col., 2016; Orlik, Hernández y Navas, 2004).

En la presente investigación se propone una herramienta a la comunidad académica que facilite la comprensión del complejo y altamente especializado lenguaje de la ciencia llamada "Química" (Sliwka, 2003; Cardellini, 2012). Intentamos lograr que el estudiante adquiera simpatía y comprensión de los contenidos temáticos introductorios a esta hermosa ciencia, atendiendo lo que menciona Córdova (1994), cuando exponía que para aprender es necesario acercarse con interés, expectativas y actitudes al conocimiento.

Nuestra propuesta combina la lúdica, la elaboración de organizadores gráficos y la retroalimentación en una estrategia didáctica que permite que los estudiantes se diviertan durante la construcción de los conocimientos de la química conceptual. La estrategia incluye actividad física, comprensión lectora, construcción de mapas conceptuales y retroalimentación. 


\section{Metodología}

Para este estudio se tomó una muestra de 120 estudiantes de segundo grado del BGC que oferta la Escuela Preparatoria Regional de Atotonilco y que cursan la unidad de aprendizaje de Química 1.

Para el contenido temático de "Química Conceptual" se diseñó esta estrategia de enseñanza-aprendizaje lúdica en la que los estudiantes se desenvuelven en un ambiente de aprendizaje distinto al aula. Durante la actividad, el alumno, corre, juega, salta, explora, lee, analiza y construye un mapa conceptual como producto final. Posteriormente se realiza una retroalimentación a los grupos tratados con la estrategia y finalmente se aplica un cuestionario para evaluar el impacto que los dos factores considerados, lúdica y retroalimentación, ejercen en el rendimiento académico de nuestros estudiantes.

Para comparar los resultados obtenidos, en el cuestionario, se diseñó un análisis de varianza de dos factores, mismo que se describe en la tabla 1:

Tabla 1. Factores aplicados a cada uno de los grupos analizados en el estudio. Fuente propia.

\begin{tabular}{c|c|c}
\hline $\begin{array}{c}\text { Factor } \\
\text { Grupo }\end{array}$ & Lúdica & Retroalimentación \\
\hline A & No & No \\
\hline B & Sí & No \\
\hline C & No & Sí \\
\hline D & Sí & Sí \\
\hline
\end{tabular}

Además la estrategia didáctica se diseñó en cuatro fases, descritas en la imagen 2, donde se indica los momentos de la actividad y se describe, posteriormente, cada uno de ellos:

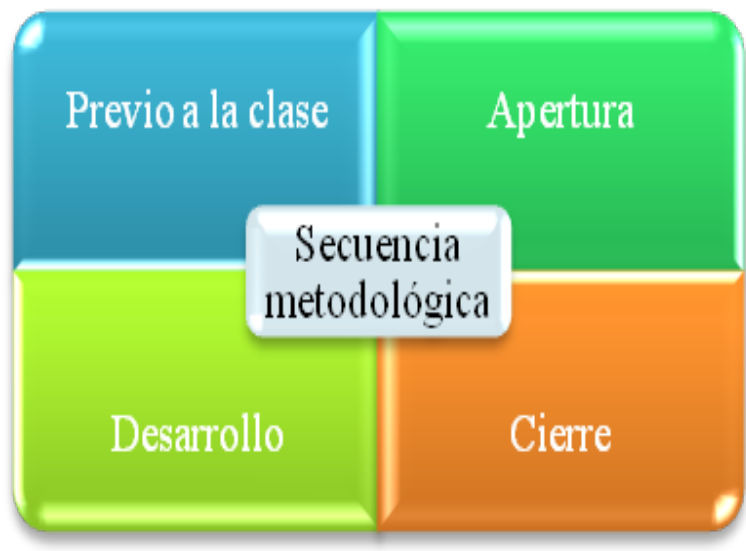

Imagen 2. Secuencia metodológica aplicada durante la investigación. Fuente propia.

\section{Previo a la clase}

El estudiante, por solicitud del profesor, debe realizar un glosario de química con conceptos de Química, nuestra propuesta se encuentra en el anexo 1.

El profesor elabora una lectura breve con los contenidos temáticos a tratar, de preferencia los que se solicitaron en el glosario (Anexo 1). Después recorta la lectura en partes según su preferencia. Puede ser por párrafos (cuando la lectura sea muy extensa), en renglones o por palabras. Una vez recortada, la lectura, los segmentos 
se meten en una bolsa, se amarra y se distribuyen en una zona de trabajo (en el aula de clases, en la cancha, en el laboratorio). Las bolsas se enumeran para que cada equipo de trabajo localice su paquete correspondiente.

\section{Apertura}

El profesor analiza junto con los estudiantes una ficha del "mapa conceptual" para recordar (toda vez que lo abordaron en la unidad de aprendizaje "habilidades para el aprendizaje") las competencias que les ayuda a desarrollar, así como la estrategia para construir dicha herramienta.

Una vez que la construcción de mapas conceptuales se ha analizado, se pide a los estudiantes que integren equipos de no más de cinco personas, se enumeran y se procede a explicar la actividad.

- Cada equipo de trabajo, con todos los integrantes, siempre, tomados de la mano, deberán buscar y encontrar un paquete que se les ha asignado con su número correspondiente.

- Cuando encuentren su paquete notarán que contiene tiras de papel con una lectura que deberán construir. Cuando se aseguren que la lectura es correcta, la deberán pegar sobre una cartulina y llevarla al profesor. Si es el docente les indica que el ejercicio es correcto, procederán al paso 3.

- A partir del glosario y la lectura, los estudiantes, construirán un mapa conceptual que contenga todos los términos abordados.

\section{Desarrollo}

Los participantes, en equipos de trabajo y tomados de la mano, salen a la cancha 0 al lugar indicado por el facilitador a buscar el paquete correspondiente. Una vez que lo encuentran, arman la frase y proceden a realizar su mapa conceptual a partir de la misma.

\section{Cierre}

El profesor presenta la lectura correcta con el auxilio de un proyector (a los grupos correspondientes) así como un mapa conceptual a manera de retroalimentación (Anexo 3).

Finalmente, se vuelve a la ficha de "mapa conceptual" y se analiza entre todos si el objetivo se logró o no, mediante la estrategia lúdica empleada, así como la retroalimentación dada.

\section{Resultados}

Se observó, a los estudiantes, realizar la actividad de manera entusiasta, alegres y colaborativa durante las tres etapas de la misma.

Al preguntarles refieren que ésta -fue una actividad dinámica, divertida, "chida"; que se habían divertido durante el desarrollo de la misma y que nunca creyeron que en la unidad de aprendizaje, química, realizarían una actividad de esta naturaleza, mencionan que son necesarias más de estas estrategias para abordar asignaturas de este tipo. 


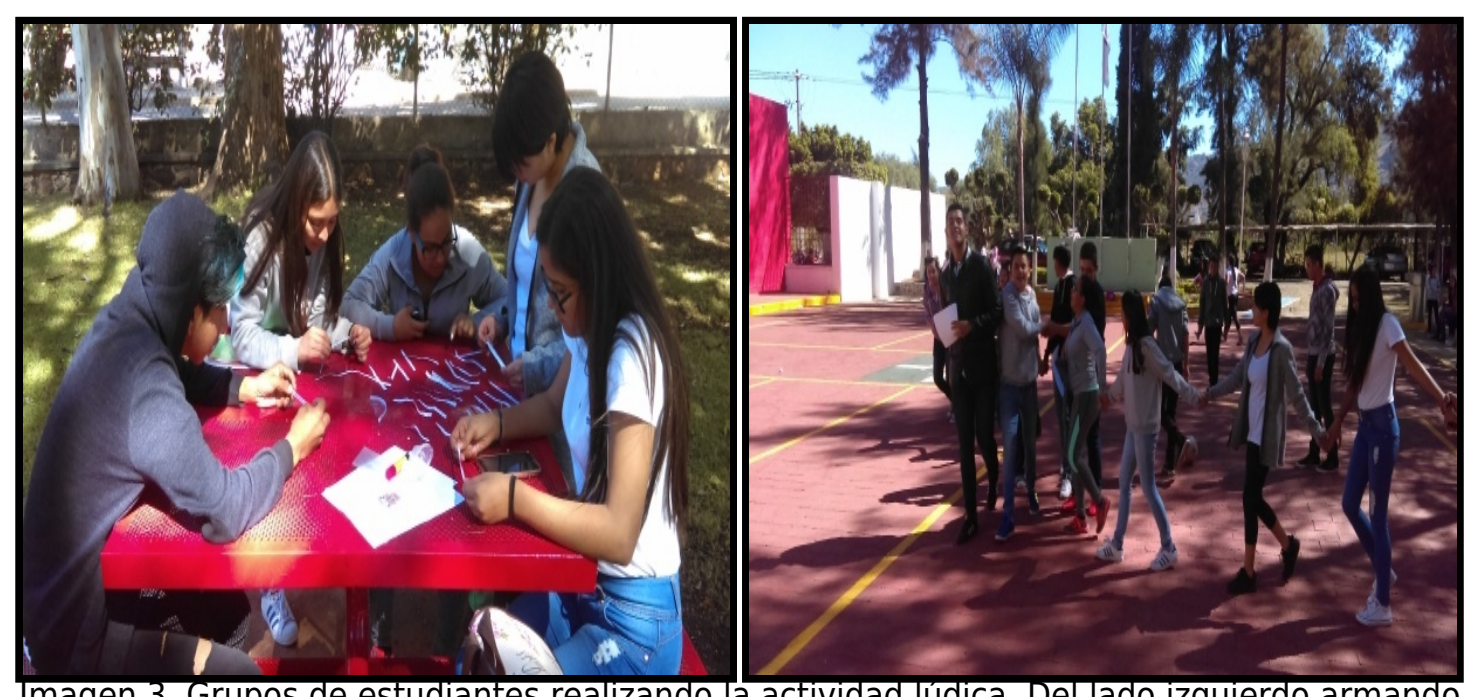

Imagen 3. Grupos de estudiantes realizando la actividad lúdica. Del lado izquierdo armando la lectura y del lado derecho buscando el paquete con la lectura recortada. Fuente propia.

Los productos entregados cumplieron con las expectativas y en la imagen 4 se exponen algunos de ellos. Son mostrados dos lecturas, en la parte superior, y dos mapas conceptuales, en la parte inferior.
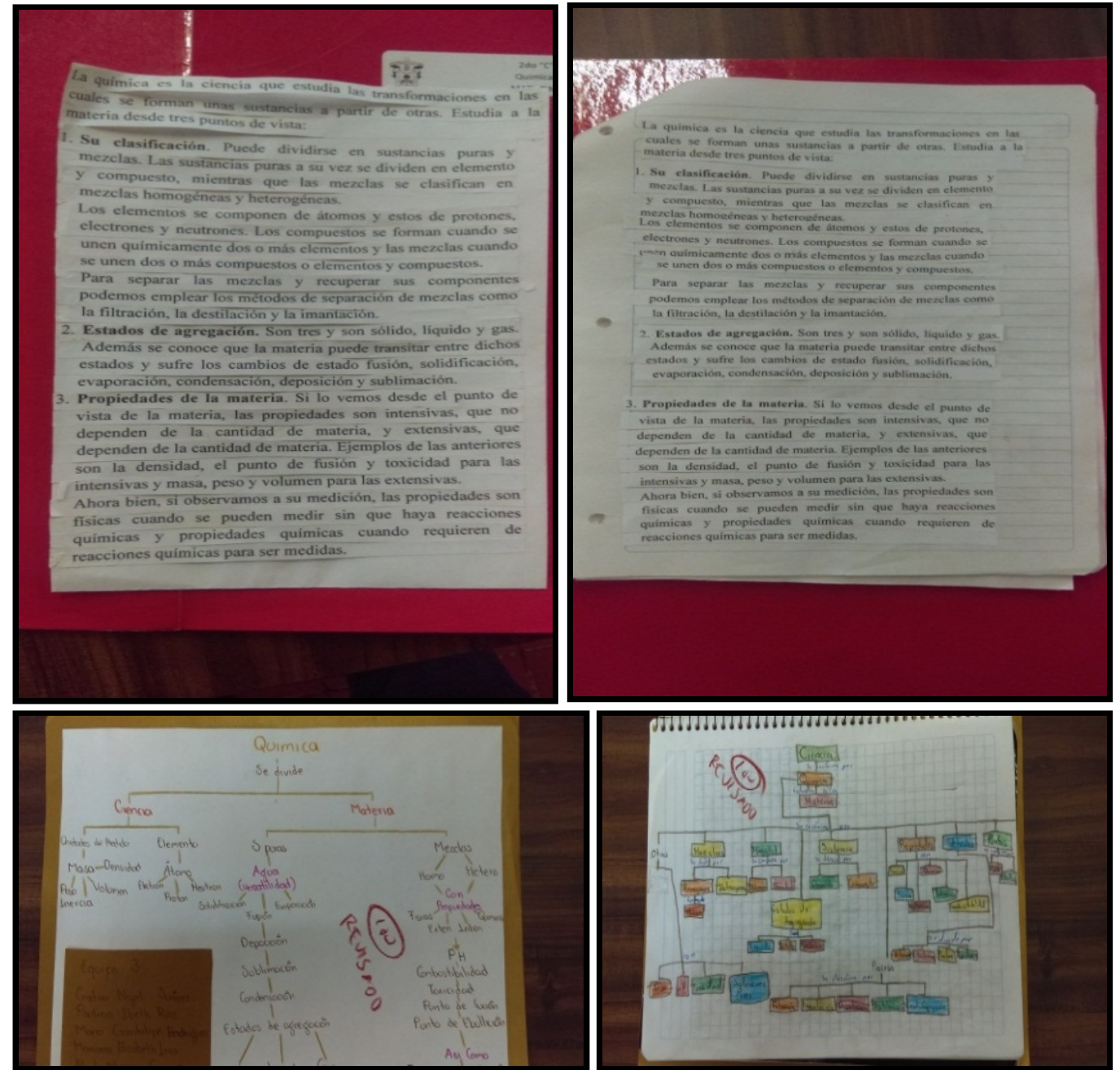

Imagen 4. Productos entregados por los estudiantes. En la parte superior se muestran dos lecturas armadas y en la parte inferior dos mapas elaborados. Fuente propia. 
Respecto a los resultados cuantitativos, mediante el análisis de varianza; ANOVA, en la imagen 5 se muestran las gráficas de medias resultantes. Del lado izquierdo se encuentra la evaluación para el factor lúdica y se observa que cuando se aplicó, los grupos tratados tuvieron un mejor desempeño a la hora de responder el cuestionario. Se observa que ambas gráficas no se traslapan, lo cual indica que existe una diferencia estadística significativa. Del lado derecho, para el factor retroalimentación, de igual manera observamos que las gráficas no se traslapan y ello indica que existe una diferencia estadística significativa, es decir, sí influye el que haya retroalimentación en la actividad analizada.

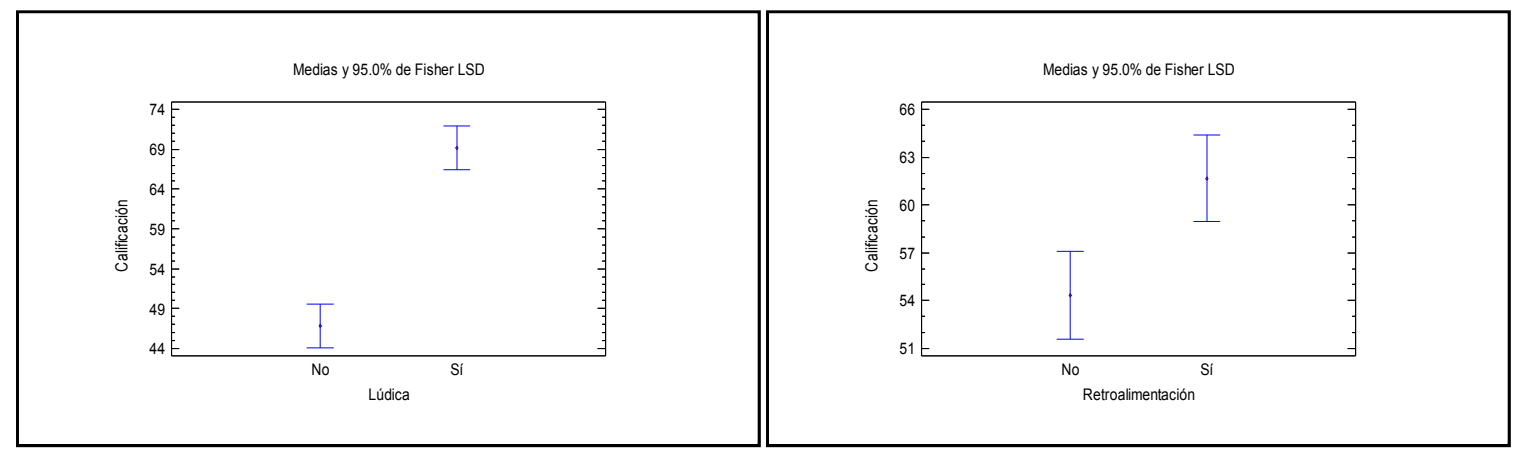

Imagen 5. Gráficas de medias para los efectos principales. Del lado izquierdo para el factor lúdica y del lado derecho el factor retroalimentación.

Observamos que, con el factor lúdica, el aumento en las calificaciones de nuestros estudiantes es de alrededor de 30 puntos. Este resultado es muy alentador debido a que en una investigación realizada, en nuestra misma escuela (Zaragoza y col., 2016), al emplear una estrategia del mismo tipo se logró un aumento máximo de 22.03 puntos en los grupos estudiados. Dicha situación permite presumir que nuestros alumnos logran una mejor estructuración de los conocimientos si los mismos son abordados a manera recreativa.

En otro estudio, González y Mora (2015) mencionan que cuando aplicaron la gamificación durante sus clases, lograron reducir de un $37 \%$ a un $14 \%$ la inasistencia a sus sesiones presenciales.

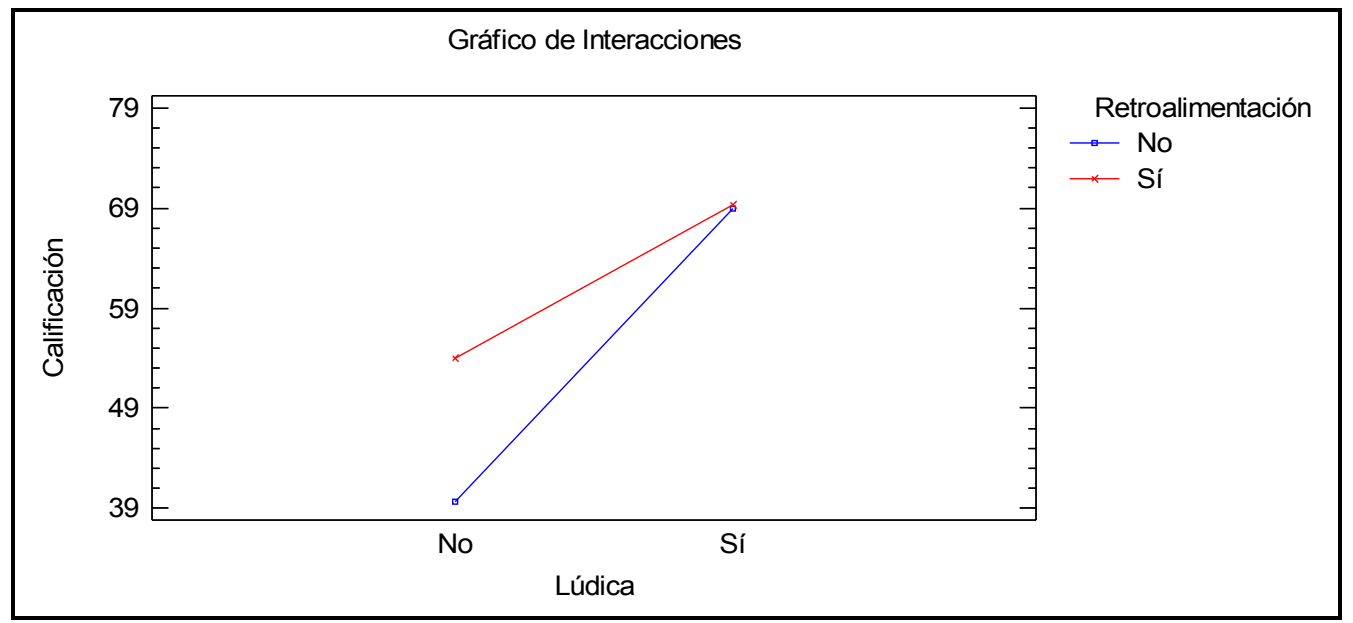

Imagen 6. Gráfica de interacciones entre los dos factores estudiados. 
Lo anterior exhorta a toda la comunidad docente a planificar las sesiones de trabajo involucrando estrategias del tipo lúdico con la finalidad de motivar a los estudiantes y que deseen acercarse a los conocimientos planteados en los objetivos establecidos.

Respecto al segundo factor, la retroalimentación, se logra un aumento de aproximadamente 15 puntos en los grupos en que se lleva a cabo, con respecto a los grupos que no tuvieron un cierre de sesión por parte del docente. Ello sugiere que si deseamos que nuestros estudiantes logren un aprendizaje mayormente estructurado, la retroalimentación es una estrategia que se puede aplicar. Es una herramienta didáctica en la que se logran reafirmar conocimientos y disolver dudas o inquietudes que los estudiantes pueden tener al finalizar una actividad.

Por último, es evidente, a partir de los resultados anteriores y la gráfica de interacciones (imagen 6), que en el grupo en que se dio la combinación de ambos factores se logró un aumento mayor que en los demás. Esto nos habla de que cuando planifiquemos una estrategia didáctica, cualquiera que sea, debemos pensar en implementar una retroalimentación para fortalecer el proceso de enseñanzaaprendizaje en nuestros estudiantes, logrando así la correcta estructuración de los conocimientos pretendidos.

\section{Conclusiones}

Con base a los resultados obtenidos, en esta y otras investigaciones mencionadas, se plantea que es necesario innovar nuestra práctica de enseñanzaaprendizaje y elaborar planeaciones académicas que incluyan actividades lúdicas. Además se sugiere que, cuando se planee la actividad, se propone realizar una retroalimentación que coadyuve a fortalecer los conocimientos esperados por los estudiantes.

Además creemos que nuestra propuesta puede ser útil no sólo en la unidad de aprendizaje, de química, sugerimos que presenta una alta transversalidad y que se puede aplicar en cualquiera otra, por lo tanto la propuesta va dirigida a toda la comunidad docente.

\section{Comentario final}

Posterior al análisis de los resultados, se retomaron los contenidos temáticos para aumentar el nivel de aprovechamiento en los grupos que obtuvieron resultados no favorables. 


\section{Referencias}

AUSUBEL, D. P; NOVAK, J. D; HANESSIAN, H. P. Psicología Educativa: un punto de vista cognoscitivo. Ciudad del Mexico: Editorial Trillas, 1976.

BRUNER, Jerome. Juego, pensamiento y lenguaje. Perspectivas, v. 16, n. 1, p. 7985, 1986.

CANNO, R. A; NEWBLE, D. A Handbook for teachers in universities and colleges. A guide to improving teaching methods. London: Routledge. 2000.

CARDELLINI, L. Chemistry: why the subject is difficult? Educación Química, 23, 305310. 2012.

CASTILLO, R. A. Construir significados al emplear mapas conceptuales soportados en las tecnologías. Télématique, v.8, n.1, p.42-51, 2009.

CÓRDOVA, F. J. L. Algunas ideas acerca del juego. Educación Química, v.5, n.2, 1994.

DROBNIC, F; GARCÍA, À; ROIG, M; GABALDÓN, S; TORRALBA, F; CAÑADA, D;

GONZÁLEZ-GROSS, M; ROMÁN, B; GUERRA, M; SEGURA, S; TIL, L; ULLOT, R; ESTEVE, I; PRAT, F. La actividad física mejora el aprendizaje y el rendimiento escolar. Esplugues de Llobregat (Barcelona): Hospital Sant Joan de Déu. 2013.

DÜRSTELER, J. Los mapas conceptuales. Revista Infovis, n. 141. 2004.

FRANCO, M. A. Diseño y evaluación del juego didáctico Química con el mundial de Brasil 2014. Educación Química, v.25, E1, p. 276-283, 2014.

GONZÁLEZ GONZÁLEZ, Carina Soledad; MORA CARREÑO, Alberto. Técnicas de gamificación aplicadas en la docencia de Ingeniería Informática. ReVision, v. 8, n. 1, 2015.

HATTIE, John; TIMPERLEY, Helen. The power of feedback. Review of educational research, v. 77, n. 1, p. 81-112, 2007.

HENRICKS, Thomas S. Play as ascending meaning: implications of a general model of play. Play contexts revisited. Stanford: Ablex Publishing Group, p. 257277, 1999.

HOLMES, Kirsten; PAPAGEORGIOU, Georgios. Good, bad and insufficient: Students' expectations, perceptions and uses of feedback. Journal of Hospitality, Leisure, Sports and Tourism Education (Pre-2012), v. 8, n. 1, p. 85, 2009.

LIEBERMAN, J. Nina. Playfulness: Its relationship to imagination and creativity. New York: Academic Press, 2014.

LIPNEVICH, A. A; SMITH, J. K. I really need feedback to learn: students' perspectives on the effectiveness of the differential feedback messages. Educational

Assessment, Evaluation and Accountability, v. 21, n. 4, p. 347, 2009. 
LOZANO MARTÍNEZ, Fernando Gustavo; TAMEZ VARGAS, Laura Adriana. Retroalimentación formativa para estudiantes de educación a distancia. RIED.

Revista Iberoamericana de Educación a Distancia, v. 17, n. 2, 2014.

MOREIRA M. Mapas conceptuales y aprendizaje significativo. 1998. Disponible en: http://www.if.ufrgs.br/ moreira/mapasesp.pdf

NOVAK J. D; GOWIN, D. B. Aprendiendo a Aprender. Santiago de Chile: Editorial Planeta Chilena. 1997.

ONTORIA, A. Mapas conceptuales, una técnica para aprender. Madrid: Nancea. 2000.

ORLIK, Y; HERNÁNDEZ, L. C; NAVAS, A. M. Sistematización de experiencias innovadoras y apropiadas sobre la enseñanza de la Ciencia y la Tecnología en el mundo y en los países CAB. Bogotá: Editorial Convenio Andrés Bello (ONCYT/CAB), 2004.

PIAGET, J; INHELDER, B. La psicología y el niño. Madrid: Ediciones Morata. 1997.

SACRISTÁN, G; PÉREZ-GÓMEZ, A. I; MARTÍNEZ, J. B; TORRES, J; ANGULO, F; ÁLVAREZ; J. M. Educar por competencias ¿qué hay de nuevo? 2a. ed. Madrid: Ediciones Morata. 2009.

SLIWKA, Hans-Richard. Reform of chemical language as a model for spelling reform. Journal of the Simplified Spelling Society, v. 32, p. 24-28, 2003.

UDG 2008. Documento Base del Bachillerato General por Competencias del Sistema de Educación Media Superior de la Universidad de Guadalajara, México. 2008.

VYGOTSKY, Lev S. The role of play in development. Mind in Society, p. 92-104, 1978.

ZARAGOZA, R. E; OROZCO, T. L; MACÍAS, G. J. O; NÚÑEZ, S. M. E; GUTIÉRREZ, G. R; HERNÁNDEZ, E. D; NAVARRO, V. C. L; DE ALBA, R. M; VILLALOBOS, D. R. M; GÓMEZ, T. N. A; CERDA, V. R. I; GUTIÉRREZ, H. A. D; PÉREZ, A. K. A., (2016). Estrategias didácticas en la enseñanza-aprendizaje: lúdica en el estudio de la nomenclatura química orgánica en alumnos de la Escuela Preparatoria Regional de Atotonilco, Educación Química, n. 27, p. 43-51. 2016. 